\title{
Afinidades biológicas de grupos pré-históricos do vale do rio Ribeira de Iguape (SP): uma análise preliminar ${ }^{1}$
}

\author{
Walter Alves Neves \\ \& Maria Mercedes Martinez Okumura ${ }^{2}$
}

\begin{abstract}
Professor do Departamento de Genética e Biologia Evolutiva - IB/USP Doutoranda do Departamento de Genética e Biologia Evolutiva - IB/USP
\end{abstract}

RESUMO: Apesar da existência de contatos entre planalto e litoral brasileiros ser atualmente quase um consenso entre os arqueólogos nacionais, não há muita certeza de como teria se dado tal contato e qual seria o fluxo entre interior e costa. O vale do Ribeira de Iguape (SP) é uma das raras regiōes do Sul-Sudeste do país onde tal comunicação seria bastante facilitada devido a peculiaridades de sua geomorfologia. Neste trabalho, apresentamos os resultados de uma análise craniométrica comparativa entre 12 esqueletos provenientes de sambaquis fluviais do vale do Ribeira datados entre 6.000 e 1.200 anos AP e 225 esqueletos oriundos de diversas séries pré-históricas brasileiras do interior e do litoral. Ao contrário do que se observa no início do Holoceno nesse vale, não há qualquer afinidade biológica entre os ribeirinhos mais tardios e os paleoíndios de Lagoa Santa ou qualquer outra série interiorana. Os grupos fluviais (ambos os sexos) associam-se aos sambaquis da costa de São Paulo e do Paraná, mostrando que houve realmente um contato considerável entre a planície costeira e o planalto, ao menos no estado de São Paulo a partir da segunda metade do Holoceno.

PALAVRAS-CHAVE: craniometria, sambaqui fluvial, Análise de Componentes Principais. 
Walter A. Neves \& Maria M. M. Okumura. Afinidades biológicas...

\section{Introdução}

O nível de contato entre planalto e litoral sul-sudeste brasileiros em tempos pré-históricos é tema polêmico entre a comunidade arqueológica nacional. Embora alguns autores tenham proposto um desenvolvimento cultural independente para o litoral e o interior (Altenfelder Silva, 1967; Laming-Emperaire, 1975), a maioria defende a idéia de contato entre tais grupos (Barreto, 1988; Schimtz et al., 1993; Schmitz, 1996; Willey, 1971). De fato, diversas hipóteses sobre a ocupação da costa brasileira já foram propostas, tais como a chegada de populaçôes vindas do planalto que teriam se instalado no litoral no meio do Holoceno ou mesmo as ocupações sazonais de um mesmo grupo no planalto e no litoral. A presença de cultura material típica do litoral sul-sudeste em sítios do planalto, como zoólitos, e da fauna marinha, como conchas de moluscos e dentes de tubarão (Barreto, 1988; Figuti et al., 2004; Tiburtius et al., 1950-1951), assim como a presença de elementos materiais idênticos em ambos os locais (costa e planalto), como é o caso de alguns tipos de cerâmica (Tradições Itararé e Tupiguarani), também contribuem para a hipótese de um contato relativamente grande entre litoral e interior na região (Araujo, 2001; Batista da Silva et al., 1990; Beck, 1971; Prous, 1979; Reis, 1980; Rohr, 1971).

Apesar disso, em termos geográficos, a comunicação entre planalto e litoral nas regiōes Sul e Sudeste brasileiras não é facilitada devido à presença da serra do Mar que, com longa escarpa e altas cristas, desde a baixada de Campos, no norte do Rio de Janeiro, até a extremidade meridional do planalto sul-brasileiro, separa de forma abrupta os dois compartimentos. O vale do rio Ribeira de Iguape, localizado no sudeste do estado de São Paulo, é um dos raros locais de ligação entre o interior e a costa na região (as outras duas áreas são o vale do Itajaí, em Santa Catarina, e a borda meridional do planalto, no Rio Grande do Sul). Tal liga- 
Revista de Antropologia, São Paulo, USP, 2005, v. 48 № 2.

ção se dá devido a características peculiares do percurso feito pelo Ribeira, que se forma no alto planalto paranaense pelos rios Assungui e Ribeirinha e, ao contrário dos demais rios da região, atravessa a serra do Mar, terminando no oceano Attântico. Dessa forma, a baixada do Ribeira é gradualmente invadida por trechos de serra que culminam nas escarpas da serra de Paranapiacaba e na serra do Mar, que a limitam a noroeste e a oeste-nordeste, respectivamente (Barreto, 1988; Petrone apud Barreto, 1988). Portanto, a ocupação dessa rara região de transição que é o vale do rio Ribeira poderia esclarecer diversas questôes sobre o contato entre o interior e a costa em tempos pré-históricos.

Os estudos arqueológicos na região do vale do Ribeira iniciaram-se no começo do século XX, com a Comissão Geográfica e Geológica do Estado de São Paulo, quando Krone (1914; 1950) mostrou ali a presença de sambaquis e "túmulos". Nessa época, Krug (1908) descreveu a escavação de um "túmulo" com fragmentos de cerâmica. Posteriormente, na década de 1930, Sakai (1981) escavou dois sambaquis fluviais na região. A partir de meados de 1970, o interesse arqueológico pelo vale do Ribeira aumentou significativamente, estimulando abordagens arqueológicas mais sistemáticas e, embora outros tipos de sítios, como sítios em abrigos, sítios a céu aberto com material lítico e cerâmico, tenham sido descritos e estudados (Barreto et al., 1982; De Blasis, 1988; 1989; Robrahn, 1988; Robrahn-González, 1999; Robrahn-González \& De Blasis, 1998), o interesse pelos sambaquis fluviais continuou muito presente (Barreto, 1988; Bartolomucci \& Eggers, 2003; 2005; BritoSchimmel et al., 2002; Carvalho et al., 2001; Collet, 1985; Collet \& Guimarães, 1977; Collet \& Loebl, 1988; Collet \& Prous, 1977; De Blasis, 2003; De Blasis et al., 1994; Figuti et al., 2000; 2001a, b; 2004; Filippini, 2004; Filippini \& Eggers, 2003; 2005; Giardini \& Eggers, 2003; 2005; Lima, 2005; Mendonça \& Gomes, 2003; Neves et al., 2005a; Petronilho et al., 2003). 
Walter A. Neves \& Maria M. M. Okumura. Afinidades biológicas...

Sambaquis fluviais são sítios arqueológicos assim denominados genericamente devido a um conteúdo expressivo de conchas de moluscos terrestres (Megalobulimus sp. e Strophocheilus sp.) e à proximidade a algum rio. Tal denominação, em contrapartida aos sítios costeiros, foi primeiramente usada na década de 1930 por Leonardos (1938 apud Lima, 1999-2000) em seu estudo "Concheiros naturais e sambaquis", no qual distingue quatro tipos de sambaquis, entre eles os fluviais, situados à beira-rio, com valvas de moluscos fluviais. Apesar de Collet e Prous (1977) descreverem tais sítios como sendo rasos (entre 50 centímetros e 1 metro de espessura) e compostos de uma terra preta com poucas conchas moídas e bolsões com grande concentração de moluscos terrestres, o termo "sambaqui fluvial" já foi utilizado para designar qualquer sítio que apresentasse conchas de moluscos terrestres em certa quantidade, não importando os demais aspectos da cultura material, a estratigrafia, a forma do sítio e o sistema de assentamento. É o caso de abrigos sob rocha que apresentam grandes concentrações de moluscos terrestres, denominados "sambaqui em abrigo sob rocha" por atravessar as serras do Mar e de Paranapiacaba, desembocando no oceano Atlântico sul (Collet, 1985), em oposição aos demais sambaquis ribeirinhos, chamados de "sambaqui ao ar livre". Não obstante o aparente contato do interior com o litoral, denunciado por diversos aspectos da cultura material dos sambaquis fluviais, Collet (1985) alerta que, "apesar da denominação 'Sambaquis' [fluviais], estas estruturas são bastante diferentes dos sambaquis stricto sensu do litoral, em função da estratigrafia mal definida e da sua composição de moluscos terrestres".

Embora no vale do Ribeira exista a maior concentração de sambaquis fluviais brasileiros, esses sítios também ocorrem em outras regiōes do país. Para o sul, há a informação de Prous (1991, p. 269) sobre a descoberta de um sambaqui formado por conchas terrestres no município de Osório, no Rio Grande do Sul. Em Santa Catarina, há um relato breve 
de Piazza (1967) sobre sambaquis fluviais em Itajaí, mas, segundo comunicação pessoal de K. Suguio, tais sítios são formados por conchas marinhas e se encontram no interior devido a uma regressão marinha recente (Prous, 1991, p. 270). Ainda em Santa Catarina, foi descrito também o sítio de Itacoara, um típico sambaqui fluvial que dista atualmente 32 quilômetros do mar e que foi datado em $1570 \pm 20$ AP (Bandeira, 2004). O sítio apresentava duas camadas distintas, a mais profunda correspondendo à parte considerada como "sambaqui fluvial". Entretanto, nessa camada, além dos moluscos fluviais, havia outros moluscos provenientes de ambientes marinhos (Amiantis purpurata, Lucina jamaicensis, Tivela brasiliana, Ostrea sp.), ossos de baleia trabalhados e dentes de cação perfurados. A cultura material encontrada nesse sítio foi descrita como típica das ocupaçôes litorâneas adjacentes (Tiburtius et al., 1950-1951). No vale do Ribeira, a indústria lítica e óssea dos sambaquis fluviais é muito semelhante à dos costeiros. Entretanto, há sítios nessa região nos quais ocorrem pontas de projéteis típicas da Tradição Umbu, o que pode ser explicado pela sobreposição de ocupações distintas (Figuti et al., 2004; Lima, 2005). Do mesmo modo, elementos da fauna litorânea também aparecem em muitos sítios do vale do Ribeira (Barreto, 1988; Figuti et al., 2004). Percebe-se, portanto, que nos sambaquis fluviais há sempre uma interação entre interior e litoral. Segundo Prous (1991, p. 272), a categoria "sambaqui fluvial" parece reunir várias realidades diferentes. $\mathrm{O}$ autor afirma que as camadas inferior e superior de Itacoara assim como os sítios do vale do Ribeira são resultantes de ocupaçōes distintas.

Apesar de o modelo predominante de povoamento da costa brasileira assumir um ou mais eixos de ocupação com base no planalto, não há evidências arqueológicas de como e quando tal processo teria se iniciado e quais rotas teriam sido usadas. Barreto (1988), por sua vez, propôs um modelo de ocupação do planalto baseado em grupos que habitavam 
Walter A. Neves \& Maria M. M. Okumura. Afinidades biológicas...

a costa e que teriam migrado para o interior, resultando nos sítios atualmente conhecidos como sambaquis fluviais. A autora afirma que os sambaquis fluviais corresponderiam a assentamentos de grupos que inicialmente teriam se instalado no litoral e que progressivamente teriam se deslocado para o interior do vale. Para aquela autora, a implantação de alguns sambaquis fluviais em áreas bem protegidas sugere a existência de pressões externas no sentido litoral-interior. Collet e Prous (1977) também afirmam que os sambaquis fluviais do vale do Ribeira marcariam uma rota temporária ou de trânsito sazonal dos grupos costeiros.

O acúmulo de conchas de moluscos terrestres e o seu aproveitamento como recurso alimentar seriam, segundo Barreto (1988), o indicador mais inequívoco de que existiria uma relação de "parentesco" entre os sambaquis fluviais e os costeiros. Para ela, a coleta de moluscos corresponderia à manutenção de um hábito adquirido no litoral, adaptado às condiçõoes ao longo do vale. A manutenção do hábito de acumular conchas longe do litoral também foi sugerida por Neves (1988), que propôs que os sambaquieiros teriam explorado também os ambientes fluviais mais próximos, tendo levado para as margens dos rios a prática do acúmulo de conchas, como seria o caso de Itacoara, em Santa Catarina.

O problema da ocupação do planalto e da costa é ainda maior se levarmos em conta a cronologia dos sítios. A diversidade encontrada nos sambaquis fluviais refere-se não somente à distribuição geográfica, mas também ao componente temporal. Há sambaquis fluviais no vale do Ribeira de Iguape datados do início do Holoceno até 1.200 anos AP, com dois hiatos, um entre 7.000-6.000 e outro entre 3.500-1.600 anos. As evidências do início do Holoceno são escassas, havendo apenas dois sítios conhecidos nesse período. Um deles, o Abrigo Maximiano, chamado por Collet (1985) de "sambaqui em abrigo sob rocha", apresenta uma datação calibrada de $9810 \pm 150$ anos AP (GIF-7493, datação não calibrada desconhecida). O outro é o sambaqui fluvial Capelinha, cuja anti- 
guidade proposta por Collet (1985) foi recentemente confirmada, por meio da datação por AMS de um dos sepultamentos em $8860 \pm 60$ AP anos AP (Beta 153988, data não calibrada). Apesar de ambos apresentarem restos de conchas, não podem ser considerados como sambaquis fluviais típicos, sendo muito provavelmente sítios multicomponenciais, frutos de diversas ocupaçôes distintas (Lima, 2005). A análise craniométrica realizada no indivíduo de Capelinha apontou forte semelhança entre ele e os paleoíndios de morfologia não mongolóide (Neves et al., 2005a).

Neste trabalho, apresentamos os resultados de uma análise morfológica comparativa efetuada com indivíduos exumados de diversos sambaquis fluviais do vale do Ribeira. Tal análise foi conduzida com base na comparação entre esses e outras séries esqueletais provenientes de sítios pré-históricos do interior e do litoral do Brasil, com o objetivo de detectar as afinidades biológicas dos grupos responsáveis pelos sambaquis fluviais da região. Trata-se, portanto, de uma análise puramente exploratória.

\section{Material e métodos}

Foram analisados 12 crânios exumados de diversos sambaquis fluviais, descritos sucintamente a seguir. As datações desses sítios encontram-se na Tabela 1, e o número de indivíduos analisados de cada sítio está nas Tabelas 2 e 3, para homens e mulheres, respectivamente.

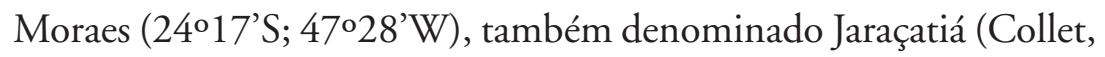
1985), localiza-se em Jacupiranga, no vale do Ribeira, na bacia do córrego do Moraes, na periferia do município de Miracatu (SP), distando do litoral 35 quilômetros em linha reta. Trata-se de uma pequena elevação cuja altura máxima é cerca de 2 metros, tendo um diâmetro de cerca de 30 metros. Predominam os artefatos líticos polidos (machados e almofarizes), além de pontas em osso e alguns raros anzóis. Elementos da fauna 
Walter A. Neves \& Maria M. M. Okumura. Afinidades biológicas...

marinha também foram encontrados, incluindo conchas de ostra e amêijoa (Lucina pectinata), dentes de tubarão e espinhos de raia trabalhados. Seixos ou almofarizes indicavam os locais de sepultamento, alguns esqueletos encontravam-se cobertos por ocre. Dos 37 sepultamentos documentados, apenas dois sepultamentos duplos foram evidenciados, sendo os restantes simples. Há sepultamentos primários e secundários. Quatro esqueletos foram datados, e suas datas não calibradas resultaram entre $5.895 \pm 45$ e $4511 \pm 32$ anos AP (Figuti et al., 2004).

Itaoca 1 (UTM 22J 0717514 / 7273961), também denominado Januário (Collet \& Guimarães, 1977; Collet \& Prous, 1977), localizase no município de Itaoca (SP). Está próximo à confluência do rio Palmital e do rio Gramado, afluentes da margem esquerda do Ribeira, implantado sobre uma pequena elevação em planície de fundo de vale, delimitado de um lado pelo rio Palmital. Atualmente, a área do sítio é a sede de uma pequena fazenda de gado. Mede cerca de $55 \mathrm{~m}$ x $45 \mathrm{~m} \mathrm{e}$ altura máxima de $150 \mathrm{~cm}$ (Barreto, 1988). Foi datado em $1460 \pm 60$ anos AP com base numa amostra de concha (Figuti et al., 2004). Collet e Guimarães (1977) realizaram uma sondagem no local, encontrando cinco sepultamentos e diversos artefatos ósseos (pontas, perfuradores, dentes trabalhados e um fragmento de anzol). Os artefatos líticos foram achados apenas nos primeiros $20 \mathrm{~cm}$ (lascas não retocadas de sílex), mas, devido a perturbações causadas por prática agrícola recente, os autores afirmam não saber a procedência estratigráfica real de tais peças. Barreto (1988) também realizou sondagens nesse sítio, evidenciando um sepultamento e diversos bolsões de concha.

Estreito 1 (24040'25"S; 4851'23"W) está localizado no município de Adrianópolis (PR), às margens do próprio Ribeira. Esse sítio foi listado por Krone (1914) e Collet (1985), tendo sido estudado posteriormente por Barreto (1988) e Figuti et al. (2004). Apresenta cerca de 40 metros de comprimento e 20 metros de largura, alcançando uma altura 
Revista de Antropologia, São Paulo, USP, 2005, v. 48 № 2.

de 2 metros. Sua delimitação a leste corresponde à barranca do rio Ribeira. Barreto (1988) descreve o material de superfície como lítico lascado em sílex e fragmentos de cerâmica. Entretanto, Figuti et al. (2004) afirmam que na indústria lítica predominam, na verdade, instrumentos de quartzo, sendo raros os artefatos de sílex. Algumas pontas em osso também foram descritas. As análises faunísticas apontam para uma predominância de peixes de água doce e de mamíferos. Seis sepultamentos foram exumados, dois deles foram datados, resultando em $3655 \pm 26 \mathrm{e}$ $4124 \pm 27$ anos AP (Figuti et al., 2004).

Pavão III (UTM 22J 0722387 / 7272285), localizado no município de Itaoca (SP), em um terraço próximo ao córrego Pavão, encontra-se sobre uma pequena elevação de fundo de vale e mede cerca de $42 \mathrm{~m} \mathrm{x}$ $56 \mathrm{~m}$, com altura máxima de $85 \mathrm{~cm}$ (Barreto, 1988). Um esqueleto humano foi datado em $1219 \pm 24$ anos AP (Figuti et al., 2004). O sítio foi estudado por Barreto (1988) que, por meio de cinco sondagens, achou grande quantidade de artefatos lascados em sílex (cuja fonte localiza-se próxima ao sítio), além de artefatos líticos polidos e fragmentos de cerâmica. À época, moradores afirmaram ter encontrado diversos sepultamentos e artefatos polidos quando da construção de uma casa sobre o sambaqui. Posteriormente, foram exumados, em caráter emergencial, sete indivíduos nesse sítio (De Blasis et al., 1994).

Pavão XVI (24039'S; 4847’ W) localiza-se no município de Itaoca (SP), em terraço plano próximo ao córrego Pavão. Situado em um local cercado por casas, não apresenta estrutura monticular, sendo um pacote arqueológico raso, com pouco menos de $40 \mathrm{~cm}$ de espessura máxima. O sítio apresenta conchas de Megalobulimus sp., ossos de animais e material lítico (a maioria lascas, mas algumas peças polidas). Um único esqueleto foi exumado a $30 \mathrm{~cm}$ de profundidade, datado em $1571 \pm 24$ anos AP (Figuti et al., 2004). 
Walter A. Neves \& Maria M. M. Okumura. Afinidades biológicas...

Os espécimes provenientes dos sambaquis fluviais acima listados foram analisados de forma comparativa em relação a amostras de referência de esqueletos pré-históricos brasileiros a partir de Análises de Componentes Principais, baseadas no conjunto de variáveis definidas por Howells (1973; 1989). A grande fragmentação de muitos dos exemplares analisados (incluindo as amostras de referência) fez com que indivíduos de sítios semelhantes (em termos geográficos, cronológicos e culturais) tivessem de ser agrupados. Pelo mesmo motivo, optou-se por trabalhar com as médias de cada série, em vez de se usar os valores de cada indivíduo. Apesar disso, ainda foi necessária a eliminação de algumas variáveis que não estavam presentes em todos os grupos. A composição de cada série mista assim como o número de indivíduos estão descritos nas Tabelas 2 e 3, respectivamente. Não há exemplares femininos exumados em sítios da Tradição Umbu que pudessem ser utilizados na análise, tampouco pôde ser composta a série mista de fluviais para esse sexo. As variáveis utilizadas para as Análises de Componentes Principais com os indivíduos do sexo masculino (47 variáveis) e do feminino (25 variáveis) separados encontram-se descritas na Tabela 4.

As Análises de Componentes Principais (Manly, 1994) foram realizadas com base apenas na forma do crânio, corrigindo-se o fator tamanho individual por meio de uma padronização $Q$ feita pela média geométrica (Darroch \& Mosiman, 1985). As afinidades biológicas dos grupos ribeirinhos do Ribeira foram exploradas pela da análise de agrupamento (cluster) aplicada a distâncias euclidianas por meio do método Ward (Ward, 1963). As distâncias foram calculadas a partir dos escores dos primeiros seis componentes, no caso dos homens, e dos primeiros quatro, no caso das mulheres. $\mathrm{O}$ número de componentes principais utilizado foi determinado por meio do valor-limite dos autovalores (Johnson \& Wichern, 2002). Esse valor-limite é determinado observando-se os primeiros valores de cada autovalor até que surja um valor que 
deixe de acrescentar informações relevantes. Dessa forma, se a porcentagem de informação relevante concentra-se do primeiro até o quarto autovalor, deve-se usar do primeiro ao quarto componente principal para o cálculo das distâncias. O uso da análise de agrupamento em vez de gráficos bidimensionais de Componentes Principais se justifica porque apenas cerca de metade da informação original foi retida pelos dois primeiros componentes, tanto no grupo masculino quanto no feminino.

\section{Resultados}

Os primeiros seis componentes principais relativos à amostra masculina explicam $88,3 \%$ da variação original da amostra. A Tabela 5 fornece a porcentagem da variação total explicada por cada um dos seis componentes principais e pelos escores obtidos para cada amostra. Esses escores foram utilizados para a construção do dendrograma da Figura 1. Verifica-se claramente nessa figura a formação de dois grandes agrupamentos: um que inclui Sumidouro e Umbu, e outro que agrega os demais grupos analisados, incluindo os provenientes de sambaquis fluviais do vale do Ribeira. Os homens de Moraes associam-se a um subgrupo formado por litoral central de São Paulo, litoral do Paraná e Guaraguaçu. Já a série mista de sítios fluviais agrupados ("Fluviais") associa-se mais fortemente ao litoral sul de São Paulo.

A análise das séries femininas mostra que os primeiros quatro componentes principais gerados explicam $87 \%$ da variação original. Os escores obtidos para cada amostra e a porcentagem da variação original explicada por cada um dos quatro componentes principais estão apresentados na Tabela 6 . Com base nesses escores, foi gerado o dendrograma da Figura 2. O resultado para os grupos femininos mostra novamente uma divisão das séries em dois grandes grupos: um deles formado por 
Walter A. Neves \& Maria M. M. Okumura. Afinidades biológicas...

litoral norte de São Paulo, interior do Paraná e Sumidouro e outro no qual se encontram as demais séries. Moraes, o único grupo representante dos sambaquis fluviais na análise sobre as mulheres, apresenta maior afinidade com um agrupamento formado por litoral sul de São Paulo, Guaraguaçu, litoral do Paraná e litoral central de São Paulo.

Além da inexistência de indivíduos oriundos de outros sambaquis fluviais que pudessem compor uma série mista feminina, a falta de séries de referência bem constituídas (isto é, provenientes de um mesmo sítio e com um número razoável de espécimes relativamente completos), que pudessem ser utilizadas nas análises para ambos os sexos, pode ter sido responsável pela variação na posição das amostras testadas nos dois dendogramas. Por exemplo, a série de Sumidouro mostra-se claramente separada das séries litorâneas e fluviais, associando-se aos indivíduos da Tradição Umbu no caso da análise masculina. Já na análise feminina, os resultados referentes a Sumidouro não são tão claros devido, provavelmente, à falta de uma outra série de morfologia não mongolóide (como é o caso da série Umbu na análise masculina) para permitir a formação de um agrupamento mais coeso. $\mathrm{Na}$ falta disso, Sumidouro associou-se aos sítios do interior do Paraná (Abrigo do Pontão e Morro dos Anjos). Apesar dessas incongruências, provavelmente de ordem amostral, há indícios que apontam para uma certa legitimidade nos resultados obtidos, seja pela estabilidade de ligação entre a série mista do litoral do Paraná e Guaraguaçu da mesma região, seja pela proximidade morfológica entre Sumidouro e Umbu no caso dos homens, afinidade esta já demonstrada em trabalho anterior (Neves et al., 2005b). 
Revista de Antropologia, São Paulo, USP, 2005, v. 48 № 2.

\section{Discussão}

Nossos resultados apóiam a idéia de um vínculo biológico entre os indivíduos dos sambaquis fluviais do vale do Ribeira e os da costa central e sul de São Paulo, assim como com os do litoral do Paraná. Tal resultado é observado tanto para homens quanto para mulheres, embora neste segundo grupo não tenha sido possível a inclusão de outra amostra fluvial que não Moraes. De qualquer modo, a série mista formada pelos homens de Itaoca 1, Estreito, Pavão III e Pavão XVI apresenta mais afinidades biológicas com a série litoral sul de São Paulo, ao passo que Moraes mostra-se mais afim morfologicamente da série litoral central de São Paulo (no caso dos homens) e do litoral sul de São Paulo (no caso das mulheres). Dado que os esqueletos ribeirinhos analisados neste trabalho são datados entre cerca de 6.000 e 1.200 anos AP, verifica-se que, pelo menos do meio do Holoceno em diante, a morfologia mongolóide estava absolutamente representada no vale do Ribeira de Iguape, ao contrário do que ocorre no início do Holoceno, período no qual prevalece a morfologia paleoamericana (identificada no sambaqui Capelinha). Infelizmente, não há esqueletos exumados de sítios da região datados do intervalo temporal entre os sítios Capelinha e Moraes (8.800 e 6.000 anos AP), de modo que não é possível saber até o momento, com precisão, quando se deu a entrada da morfologia mongolóide no vale do Ribeira. Uma vez que todas as populaçôes sambaquieiras do litoral sul e sudeste brasileiros são tipicamente mongolóides, é possível conjecturar que reside ali a origem dos construtores dos sambaquis fluviais do sul do estado de São Paulo.

Apesar dos sambaquis fluviais serem conhecidos desde o início do século passado e de terem sido estudados sistematicamente a partir da segunda metade do século XX, estudos morfológicos sobre esqueletos exumados desses sítios são raros. Nesse sentido, o presente trabalho deve 
Walter A. Neves \& Maria M. M. Okumura. Afinidades biológicas...

ser visto como uma das primeiras aproximações ao problema, aproximação esta baseada, infelizmente, em uma amostra mais do que imperfeita. Em relação aos demais sambaquis fluviais brasileiros, apenas o sítio catarinense de Itacoara, se é que pode ser considerado como tal, foi investigado com base numa análise morfológica envolvendo caracteres não métricos cranianos. Nessa análise, foi verificada uma grande semelhança entre os indivíduos provenientes de Itacoara e aqueles dos sambaquis clássicos do litoral norte de Santa Catarina (Neves, 1988). A semelhança morfológica por nós encontrada entre indivíduos exumados no vale do Ribeira e no litoral centro-sul de São Paulo, entretanto, parece não ter sido verificada em outros estudos. Análises de caracteres cranianos não métricos, por exemplo, apontaram para uma diferença considerável entre os grupos de sambaquis fluviais e costeiros (Filippini, 2004; Filippini \& Eggers, 2003; 2005). Entretanto, tais resultados devem ser aceitos com certa reserva, entre outras razões, pelo fato de terem sido incluídos neles apenas dois sambaquis litorâneos de São Paulo e um de Santa Catarina como séries comparativas. Além disso, entre os espécimes provenientes de sambaquis fluviais foi incluído o de Capelinha, sabidamente não mongolóide. Outro estudo recente (Giardini \& Eggers, 2003; 2005; Bartolomucci, 2006) envolvendo marcadores não métricos dentários apontou para uma proximidade biológica entre os sambaquis fluviais do Ribeira e os da costa do Paraná (sambaquis do litoral de São Paulo não foram incluídos na análise, apenas sambaquis do Paraná, norte e sul de Santa Catarina, incluindo Itacoara).

A existência de afinidades biológicas entre os indivíduos provenientes de sambaquis costeiros e fluviais tardios do estado de São Paulo suporta, ainda que muito preliminarmente, a hipótese de um fluxo gênico acentuado entre planalto e litoral no extremo sul do estado de São Paulo, confluindo com a semelhança da cultura material e, obviamente, com o próprio hábito de acumular conchas no espaço residencial. Além do 
mais, conforme dito anteriormente, o vale do Ribeira é uma das raras regiōes no Brasil onde a transição da costa para o interior dá-se de modo não abrupto, de modo que uma relação direta entre tais grupos teria sido ali imensamente facilitada.

Tabela 1: Datações dos sítios estudados, efetuadas em ossos humanos (exceto Itaoca 1, efetuada em conchas)

\begin{tabular}{|c|c|c|c|c|}
\hline Sítio & Unidade & $\begin{array}{l}\text { Profundidade } \\
\qquad(\mathrm{cm})\end{array}$ & $\begin{array}{l}\text { Idade não } \\
\text { calibrada }\end{array}$ & $\begin{array}{c}\text { № de } \\
\text { laboratório }\end{array}$ \\
\hline Moraes (Jaraçatiá) & Sep 25 & 25 & $4511 \pm 32$ & KIA* 20844 \\
\hline Moraes (Jaraçatiá) & Sep 35 & 35 & $5420 \pm 30$ & KIA 20843 \\
\hline Moraes (Jaraçatiá) & Sep 5 & 100 & $4985 \pm 35$ & KIA 15562 \\
\hline Moraes (Jaraçatiá) & Sep 13 & 130 & $5895 \pm 45$ & KIA 15561 \\
\hline Itaoca 1 (Januário) & S1 & $20-30$ & $1460 \pm 60$ & Beta** $^{* *} 178128$ \\
\hline Estreito & Sep 1 & 25 & $3655 \pm 26$ & KIA 20845 \\
\hline Estreito & Sep 6 & 130 & $4124 \pm 27$ & KIA 20846 \\
\hline Pavão XVI & Sep 1 & 30 & $1571 \pm 24$ & KIA 20842 \\
\hline Pavão III & Sep 1 & 20 & $1219 \pm 24$ & KIA 20840 \\
\hline
\end{tabular}

* KIA: Leibniz-Labor for Radiometric Dating and Isotope Research, ChristianAlbrechts-University Kiel, Alemanha.

** Beta: Beta Analytic., Inc. 


\section{Tabela 2: Amostras masculinas utilizadas na análise}

\begin{tabular}{|c|c|c|c|c|c|}
\hline Série & $\begin{array}{l}\text { Sítios arqueológicos } \\
\text { que compóem a série }\end{array}$ & Região & $\begin{array}{c}\text { Datação } \\
\text { mais antiga }\end{array}$ & $\begin{array}{l}\text { № por } \\
\text { sítio }\end{array}$ & $\begin{array}{c}\text { № } \\
\text { total }\end{array}$ \\
\hline Moraes & Idem série & Vale do Ribeira de Iguape, SP & $5895 \pm 45$ & - & 4 \\
\hline \multirow[t]{4}{*}{ Fluviais } & Itaoca 1 & Vale do Ribeira de Iguape, SP & $1460 \pm 60$ & 1 & 5 \\
\hline & Estreito 1 & Vale do Ribeira de Iguape, PR & $4124 \pm 27$ & 1 & \\
\hline & Pavão III & Vale do Ribeira de Iguape, SP & $1219 \pm 24$ & 2 & \\
\hline & Pavão XVI & Vale do Ribeira de Iguape, SP & $1571 \pm 24$ & 1 & \\
\hline Sumidouro & Idem série & Lagoa Santa, MG & $>8,000$ & - & 17 \\
\hline Umbu & Idem série & RS & - & - & 3 \\
\hline Est. Comprido & Idem série & Prudentópolis, PR & - & - & 4 \\
\hline \multirow[t]{2}{*}{ Interior PR } & Abrigo do Pontão & Sengés, PR & - & 2 & 3 \\
\hline & Morro dos Anjos & Adrianópolis, PR & - & 1 & \\
\hline Guaraguaçu & Idem série & Paranaguá, PR & $4200 \pm 200$ & - & 20 \\
\hline \multirow[t]{10}{*}{ Litoral PR } & Matinhos & Matinhos, PR & $2750 \pm 250$ & 10 & 25 \\
\hline & Centenário & Paranaguá, PR & - & 3 & \\
\hline & Macedo & Paranaguá, PR & $3677 \pm 61$ & 3 & \\
\hline & Ilha dos Ratos & Guaratuba, PR & $1540 \pm 150$ & 2 & \\
\hline & Araújo II & Guaratuba, PR & - & 1 & \\
\hline & Guaratuba (regiāo) & Guaratuba, PR & - & 1 & \\
\hline & Rio Laranjeiras & Guaratuba, PR & - & 1 & \\
\hline & Toral 51 & Paranaguá, PR & - & 1 & \\
\hline & Ilha Rasa (região) & Guaraqueçaba, PR & - & 1 & \\
\hline & Ponta do Goulart (região) & Antonina, PR & - & 2 & \\
\hline
\end{tabular}


Revista de Antropologia, São Paulo, USP, 2005, v. 48 no 2.

\begin{tabular}{|c|c|c|c|c|}
\hline Litoral norte & Tenório & Ubatuba, SP & $1875 \pm 90$ & 3 \\
\hline SP & Mar Virado & Ilha do Mar Virado, SP & $2640 \pm 70$ & 2 \\
\hline Litoral central & Piaçaguera & Cubatão, SP & $4930 \pm 110$ & 17 \\
\hline \multirow[t]{6}{*}{ SP } & Ilha de Santo Amaro & Ilha de Santo Amaro, SP & - & 12 \\
\hline & Buracão & Ilha de Santo Amaro, SP & $2050 \pm 100$ & 6 \\
\hline & Maratuá & Ilha de Santo Amaro, SP & $3865 \pm 95$ & 4 \\
\hline & Casqueirinha & Santos, SP & - & 1 \\
\hline & Santos (região) & Santos, SP & - & 1 \\
\hline & Cosipa 4 & Santos, SP & $2590 \pm 80$ & 1 \\
\hline Litoral sul & Boguassu & Ilha Comprida, SP & - & 4 \\
\hline \multirow[t]{4}{*}{ SP } & Iguape (região) & Iguape, SP & - & 4 \\
\hline & Brocoanha II & Baixada Cananéia-Iguape, SP & - & 2 \\
\hline & Boa Vista & Ilha Comprida, SP & - & 1 \\
\hline & Cananéia (região) & Cananéia, SP & - & 1 \\
\hline
\end{tabular}

Total

Na segunda coluna, nomes seguidos da palavra "região" entre parênteses indicam indivíduos provenientes de sambaquis costeiros de determinada região, porém, sem procedência exata de sítio arqueológico. 


\section{Tabela 3: Amostras femininas utilizadas na análise}

\begin{tabular}{|c|c|c|c|c|c|}
\hline \multicolumn{3}{|c|}{$\begin{array}{l}\text { Sítios arqueológicos } \\
\text { que compóem a série }\end{array}$} & \multirow{2}{*}{$\begin{array}{c}\text { Datação } \\
\text { mais antiga } \\
5895 \pm 45\end{array}$} & \multirow{2}{*}{$\begin{array}{l}\text { № por } \\
\text { sítio } \\
-\end{array}$} & \multirow{2}{*}{$\begin{array}{c}\text { № } \\
\text { total } \\
3\end{array}$} \\
\hline Moraes & Idem série & Jale do Ribeira de Iguape, SP & & & \\
\hline Sumidouro & Idem série & Lagoa Santa, MG & $>8,000$ & - & 13 \\
\hline Estirão Comprido & Idem série & Prudentópolis, PR & - & - & 3 \\
\hline \multirow[t]{2}{*}{ Interior PR } & Abrigo do Pontão & Sengés, PR & - & 4 & 7 \\
\hline & Morro dos Anjos & Adrianópolis, PR & - & 3 & \\
\hline Guaraguaçu & Idem série & Paranaguá, PR & $4200 \pm 200$ & - & 19 \\
\hline \multirow[t]{7}{*}{ Litoral PR } & Matinhos & Matinhos, PR & $2750 \pm 250$ & 11 & 19 \\
\hline & Ilha dos Ratos & Guaratuba, PR & $1540 \pm 150$ & 3 & \\
\hline & Rio da Praia & Guaratuba, PR & - & 1 & \\
\hline & Boguassu & Guaratuba, PR & - & 1 & \\
\hline & Macedo & Paranaguá, PR & $3677 \pm 61$ & 1 & \\
\hline & a do Veríssimo (região) & Paranaguá, PR & - & 1 & \\
\hline & Saquarema & Morretes, PR & $4438 \pm 89$ & 1 & \\
\hline Litoral norte SP & Tenório & Ubatuba, SP & $1875 \pm 90$ & - & 7 \\
\hline \multirow[t]{4}{*}{ Litoral central SP } & Buracão & Ilha de S. Amaro, SP & $2050 \pm 100$ & 5 & 15 \\
\hline & Maratuá & Ilha de S. Amaro, SP & $3865 \pm 95$ & 4 & \\
\hline & le Santo Amaro (região) & Ilha de S. Amaro, SP & - & 4 & \\
\hline & Piaçaguera & Cubatão, SP & $4930 \pm 110$ & 2 & \\
\hline \multirow[t]{4}{*}{ Litoral Sul SP } & Boguassu & Ilha Comprida, SP & - & 5 & 11 \\
\hline & Brocoanha II & Baixada Cananéia-Iguape, SP & - & 3 & \\
\hline & Boa Vista & Ilha Comprida, SP & - & 2 & \\
\hline & Iguape (regiāo) & Iguape, SP & - & 1 & \\
\hline Total & & - & - & & 97 \\
\hline
\end{tabular}

Na segunda coluna, nomes seguidos da palavra "região" entre parênteses indicam indivíduos provenientes de sambaquis costeiros de determinada região, porém, sem procedência exata de sítio arqueológico. 


\section{Tabela 4: Variáveis usadas na Análise de Componentes Principais}

O asterisco e o traço indicam, respectivamente, a presença e a ausência da variável no caso de cada parcela sexual.

\section{Variável Descrição}

GOL Comprimento da glabela ao occipital

NOL Comprimento do nasion ao occipital

BNL Comprimento basion-nasion

$\mathrm{BBH} \quad$ Comprimento basion-bregma

$\mathrm{XCB} \quad$ Largura máxima craniana

XFB Largura máxima frontal

STB Largura bistefânica

ZYB Largura bizigomática

AUB Largura biauricular

WCB Largura craniana mínima

ASB Largura biasterionica

BPL Comprimento basion-prosthion

$\mathrm{NPH}$ Altura nasion-prosthion

$\mathrm{NLH} \quad$ Altura nasal

$\mathrm{OBH} \quad$ Altura da órbita

OBB Largura da órbita

JUB Largura bijugal

NLB Largura nasal

$\mathrm{MAB}$ Largura máxima alveolar

ZMB Largura bimaxilar

SSS Subtense (zmb) no subspinale

FMB Largura bifrontal

NAS Subtense ( $\mathrm{fmb}$ ) no nasion

EKB Largura biorbital
Masc. Fem.

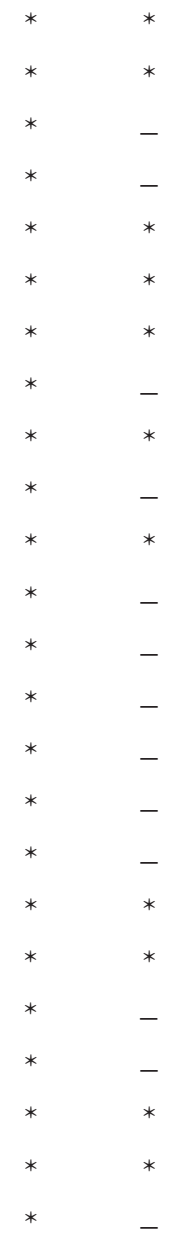


DKS Subtense (ekb) no dakryon

DKB Largura entre dk-dk

WNB Largura mínima nasal

IML Comprimento inferior do malar

XML Comprimento máximo do malar

MLS Subtense (xml) máxima no ângulo do malar

WMH Altura mínima do malar

FOL Comprimento do forame magno

FRC Corda frontal

FRS Projeção máxima da convexidade do osso frontal, no eixo formado pela corda frontal

FRF Distância entre o nasion e a projeção máxima da convexidade do osso frontal

PAC Corda parietal

PAS Projeção máxima da convexidade do osso parietal, no eixo formado pela corda parietal

PAF Distância entre o bregma e a projeção máxima da convexidade do osso parietal

OCC Corda occipital

OCS Projeção máxima da convexidade do osso occipital, no eixo formado pela corda occipital

OCF Distância entre o lambda e a projeção máxima da convexidade do osso occipital

VRR Maior distância perpendicular ao eixo transmeatal e o eixo sagital médio

NAR Distância perpendicular entre o eixo transmeatal e o nasion

SSR Distância perpendicular entre o eixo transmeatal e o subspinale 
RR Distância perpendicular entre o eixo transmeatal e o prosthion

DKR Distância perpendicular entre o eixo transmeatal e o dacryon

ZOR Distância perpendicular entre o eixo transmeatal e o zigoorbitale

FMR Distância perpendicular entre o eixo transmeatal e o frontomalare

EKR Distância perpendicular entre o eixo transmeatal e o ectoconchion

ZMR Distância perpendicular entre o eixo transmeatal e o zigomaxilar anterior

AVR Distância perpendicular entre o eixo transmeatal e o ponto mais anterior do primeiro molar

BRR Distância perpendicular entre o eixo transmeatal e o bregma

LAR Distância perpendicular entre o eixo transmeatal e o lambda

OSR Distância perpendicular entre o eixo transmeatal e o opisthion

BAR Distância perpendicular entre o eixo transmeatal e o basion 
Walter A. Neves \& Maria M. M. Okumura. Afinidades biológicas...

\section{Tabela 5: Autovalores e porcentagem da variação explicada pelos seis primeiros componentes principais e pelos escores para cada série masculina}

$\begin{array}{lcccccc}\text { Série } & \text { Fator 1 } & \text { Fator 2 } & \text { Fator 3 } & \text { Fator 4 } & \text { Fator 5 } & \text { Fator 6 } \\ \text { Autovalores } & 0,018246 & 0,013063 & 0,008984 & 0,006438 & 0,004890 & 0,004786 \\ \% & 28,72 & 20,56 & 14,14 & 10,13 & 7,70 & 7,53 \\ \text { Moraes } & -0,076605 & -0,071517 & 0,101201 & 0,092552 & 0,103865 & 0,012046 \\ \text { Fluviais } & 0,039629 & 0,128574 & -0,002762 & 0,084621 & -0,057590 & 0,164572 \\ \text { Sumidouro } & 0,287084 & -0,023250 & -0,062170 & -0,087052 & 0,000285 & -0,007611 \\ \text { Umbu } & 0,203997 & 0,031488 & 0,101704 & 0,052363 & -0,048123 & -0,067946 \\ \text { Interior PR } & 0,004702 & -0,142746 & -0,194929 & 0,120278 & 0,020457 & -0,034070 \\ \text { Estirāo Comprido } & -0,130987 & -0,024470 & 0,060816 & 0,022009 & -0,127223 & -0,071777 \\ \text { Guaraguaçu } & -0,008876 & -0,036538 & 0,105501 & -0,011551 & 0,051836 & 0,001999 \\ \text { Litoral PR } & -0,035693 & -0,036497 & 0,050541 & -0,085163 & 0,034591 & -0,002350 \\ \text { Litoral norte SP } & -0,138631 & -0,111249 & -0,046032 & -0,095279 & -0,079493 & 0,043705 \\ \text { Litoral central SP } & -0,035002 & 0,019666 & -0,030530 & -0,082524 & 0,067044 & 0,039802 \\ \text { Litoral sul SP } & -0,109619 & 0,266540 & -0,083341 & -0,010254 & 0,034351 & -0,078370\end{array}$




\section{Tabela 6: Autovalores e porcentagem da variação explicada pelos cinco primeiros componentes principais e pelos escores para cada série feminina}

$\begin{array}{lcccc}\text { Série } & \text { Fator } 1 & \text { Fator } 2 & \text { Fator } 3 & \text { Fator 4 } \\ \text { Autovalores } & 0,013045 & 0,010018 & 0,005443 & 0,004311 \\ \% & 34,59780 & 26,56840 & 14,43462 & 11,43407 \\ \text { Moraes } & 0,141333 & 0,026904 & -0,120041 & 0,072098 \\ \text { Sumidouro } & -0,216224 & 0,039803 & 0,053985 & 0,052338 \\ \text { Estirão Comprido } & -0,060158 & -0,227853 & -0,061580 & -0,016673 \\ \text { Interior PR } & -0,082415 & 0,103510 & -0,054733 & 0,061039 \\ \text { Guaraguaçu } & 0,008042 & -0,066392 & 0,060830 & 0,011952 \\ \text { Litoral PR } & 0,071615 & -0,013830 & 0,062765 & -0,021157 \\ \text { Litoral norte SP } & -0,038318 & 0,097179 & -0,056792 & -0,143544 \\ \text { Litoral central SP } & 0,037812 & 0,007473 & 0,027077 & -0,032768 \\ \text { Litoral sul SP } & 0,138313 & 0,033206 & 0,088489 & 0,016717\end{array}$


Walter A. Neves \& Maria M. M. Okumura. Afinidades biológicas...

Figura 1: Dendrograma (método Ward) entre as séries masculinas incluídas no trabalho. A distância de ligação é baseada nos primeiros seis Componentes Principais

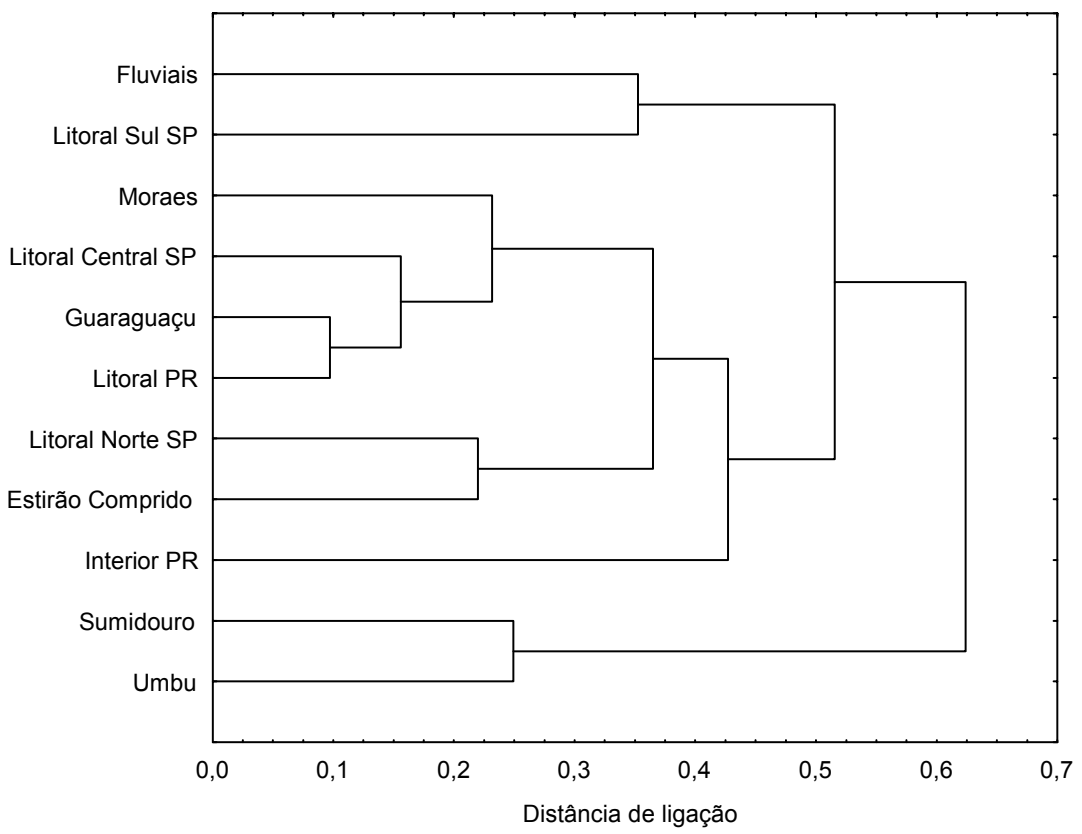


Revista de Antropologia, São Paulo, USP, 2005, v. 48 № 2.

Figura 2: Dendrograma (método Ward) entre as séries femininas incluídas no trabalho. A distância de ligação é baseada nos primeiros seis Componentes Principais

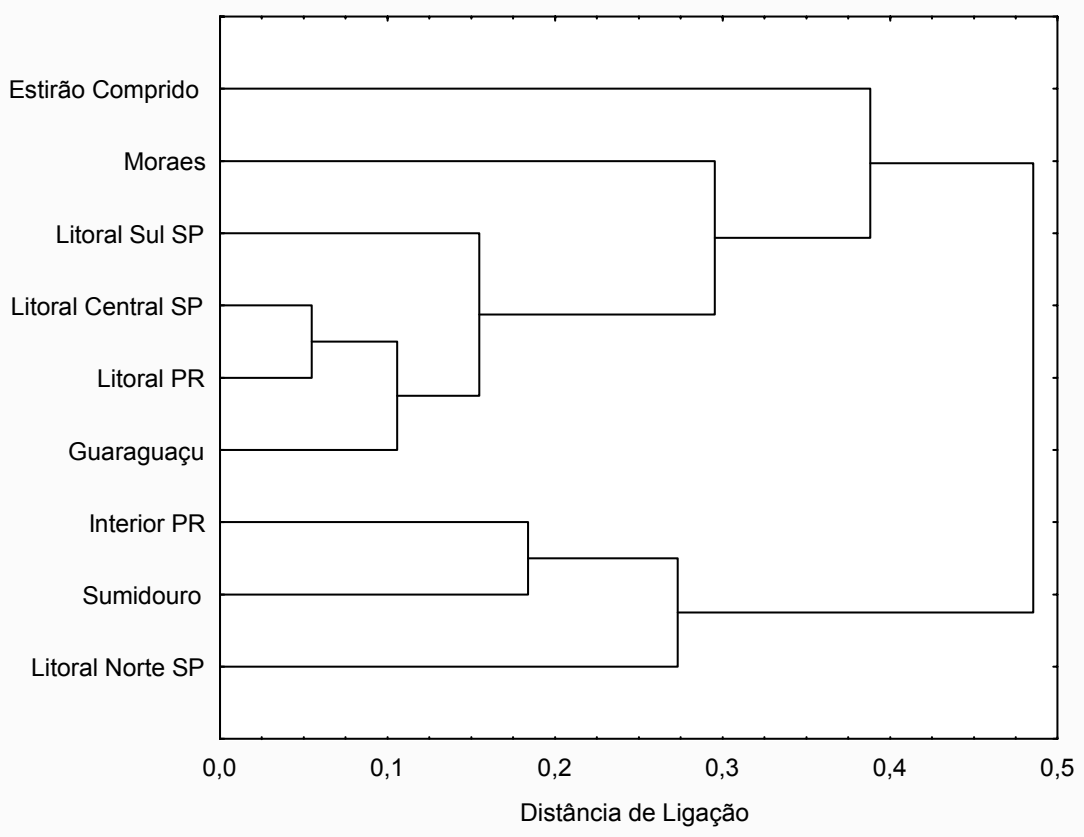




\section{Notas}

1 Agradecemos a Hilton Pereira da Silva, Sheila Mendonça de Souza e Cláudia Rodrigues-Carvalho do Museu Nacional (UFRJ-RJ), Murillo Marx, Levi Figuti, Paulo Dantas De Blasis e Dorath Pinto Uchôa do Museu de Arqueologia e Etnologia (USP-SP), Sabine Eggers do Instituto de Biociências (USP-SP), Cláudia Inês Parellada do Museu Paranaense (UFPR-PR), Igor Chmyz do Centro de Estudos e Pesquisas Arqueológicas (UFPR-PR), Ana Luiza Fayet Salla e Patrícia Gaulier do Museu de Arqueologia e Etnologia (UFPR-PR), Dione da Rocha Bandeira, Adriana Maria Pereira dos Santos e Maria Cristina Alves do Museu Arqueológico de Sambaqui de Joinville (MASJ-SC), André Jacobus do Museu Arqueológico do Rio Grande do Sul (MARSUL-RS) e Kim Aaris-Sørensen do Zoological Museum (University of Copenhagem) pelo acesso às coleçôes de esqueletos humanos necessárias ao presente trabalho. Esta pesquisa foi financiada pela Fapesp através dos processos 04/01321-6 (WAN) e 02/13441-0 (MMMO). Walter Neves é bolsista de produtividade científica do CNPq (processo 305918/00-85).

2 É também curadora-pesquisadora do Leverhulme Centre for Human Evolutionary Studies (LCHES) na Universidade de Cambridge, Reino Unido.

\section{Bibliografia}

ALTENFELDER SILVA, F.

1967 "Informes preliminares sobre a arqueologia de Rio Claro. PRONAPA 1: Resultados preliminares do primeiro ano (1965/66)", Publicaçōes Avulsas, Museu Paraense Emílio Goeldi, vol. 6.

ARAUJO, A. G. M.

2001 Teoria e método em arqueologia regional: um estudo de caso no alto Paranapanema, Estado de São Paulo, tese de doutorado, São Paulo, Universidade de São Paulo.

BANDEIRA, D. R.

2004 Ceramistas pré-coloniais da Baía da Babitonga, SC - arqueologia e etnicidade, tese de doutorado, Campinas, Universidade Estadual de Campinas. 
BARRETO, C. N. G. B.

1988

A ocupação pré-colonial do Vale do Ribeira de Iguape, SP: os sitios concheiros do médio curso, tese de doutorado, São Paulo, Universidade de São Paulo.

BARRETO, C. N. G. B.; DE BLASIS, P. A. D.; DIAS NETO, C. M.; KARMANN, I.; FERREIRA LINO, C. \& ROBRAHN, E. M.

1982 "Abismo Ponta de Flecha: um projeto arqueológico, paleontológico e geológico no médio vale do Ribeira de Iguape, São Paulo", Revista de Pré-História, 3, p. $195-215$.

BARTOLOMUCCI, L. B. G.

2006 Variabilidade biológica entre sambaquieiros: um estudo de morfologia dentária, dissertação de mestrado, São Paulo, Universidade de São Paulo.

BARTOLOMUCCI, R. \& EGGERS, S.

2003 "Preservação óssea: uma questão arqueológica", Resumos do XII Congresso da Sociedade de Arqueologia Brasileira, São Paulo.

2005 "Human taphonomic analyses applied to the fluvial shellmounds of the Valley of Ribeira de Iguape, São Paulo", Abstracts 1st Paleopathology Association Meeting in South America, Rio de Janeiro.

BATISTA DA SILVA, S.; SCHMITZ, P. I.; ROGGE, J. H.; DE MASI, M. A. N. \& JACOBUS, A. L.

1990 "Escavações arqueológicas do Pe. João Alfredo Rohr, s. j. - o sítio arqueológico da praia da Tapera: um assentamento Itararé e Tupiguarani”, Pesquisas (Antropologia), vol. 45, p. 1-210.

BECK, A.

1971

"Grupos cerâmicos do litoral de Santa Catarina. Fase Rio Lessa e Fase Enseada”, Anais do Museu de Antropologia UFSC, vol. 4, p. 25-29.

BRITO-SCHIMMEL, P.; PORSANI, J. L.; FIGUTI, L.; DE BLASIS, P. A. D.

2002 "Geophysical methods applied in archaeology: first results from Capelinha fluvial sambaqui, Cajati - SP, Brasil”, Revista do Museu de Arqueologia e Etnologia, vol. 12 , p. 43-54. 
Walter A. Neves \& Maria M. M. Okumura. Afinidades biológicas...

CARVALHO, D. S.; MENDONÇA, C. A.; ROCHA, E. B.; ROMERO, T. M.; YOKOYAMA, L. M. L.; GOMES, M. J.

2001 "Investigaçôes geofísicas no sambaqui fluvial dos Moraes, Miracatu, SP", Resumos do VI Congresso Internacional da Sociedade Brasileira de Geofisica, Salvador.

COLLET, G. C.

1985 "Novas informaçôes sobre os sambaquis fluviais do Estado de São Paulo", Arquivos do Museu de História Natural, Belo Horizonte, vol. 10, p. 311-24.

COLLET, G. C. \& GUIMARĀES, C. M.

1977 "Resultado da sondagem do sambaqui Januário", Arquivos do Museu de História Natural, Belo Horizonte, vol. 2, p. 36-50.

COLLET, G. \& PROUS, A.

1977 "Primeiro informe sobre os sambaquis fluviais da região de Itaoca (SP)", Arquivos do Museu de História Natural, Belo Horizonte, vol. 2, p. 31-35.

COLLET, G. C. \& LOEBL, E. 1988 "Informaçóes sobre sambaquis fluviais do Estado de São Paulo", Anuário Staden (Estudos Brasileiros), vol. 36.

DARROCH, J. N. \& MOSIMANN, J. E.

1985 "Canonical and principal components of shape", Biometrika, vol. 72(2), p. 241-52.

DE BLASIS, P. A. D. A ocupação pré-colonial do vale do Ribeira de Iguape, SP: os sítios líticos do médio curso, dissertação de mestrado, São Paulo, Universidade de São Paulo.

1989 "A indústria dos sítios líticos do médio Vale do Ribeira de Iguape: um ensaio tipológico”, Revista de Pré-História, São Paulo, 7, p. 89-111.

2003

"Os sambaquis fluviais do vale do Ribeira de Iguape: evidências intra e extrasítio", Resumos do XII Congresso da Sociedade de Arqueologia Brasileira, São Paulo. 
DE BLASIS, P. A. D.; PIEDADE, S. C.; MORALES, W. F.

1994 "Algumas considerações sobre os sambaquis fluviais do médio Ribeira, SP", Revista do Museu de Arqueologia e Etnologia, São Paulo, vol. 4, p. 218-19.

FIGUTI, L.; DE BLASIS, P. A. D.; MENDONÇA, C. A.

2000 "A aplicação de métodos geofísicos nos sambaquis fluviais do vale do Ribeira de Iguape, São Paulo, Brasil”, Resumos da II Reunião de Teoria Arqueológica da América do Sul, Olavarria, Argentina.

2001a "Geophysical and archaeological investigations on inland riverine sambaquis, São Paulo, Brasil”, Abstracts 66th Annual Meeting of Society for American Archaeology, New Orleans.

FIGUTI, L.; DE BLASIS, P. A. D.; PLENS, C. R.; EGGERS, S.

2001b "Um sepultamento de 9.000 anos: cultura, saúde e atividade", Resumos do XI Congresso da Sociedade de Arqueologia Brasileira, Rio de Janeiro.

FIGUTI, L.; EGGERS, S.; MENDONÇA, C. A.; PORSANI, J. L.; ROCHA, E. B.; DE BLASIS, P. A. D.; BISSA, W. M.

2004

Investigaçôes arqueológicas e geofisicas dos sambaquis fluviais do vale do Ribeira de Iguape, Estado de São Paulo. Museu de Arqueologia e Etnologia, USP. Relatório Final de Atividades de Projeto Temático, processo FAPESP nº 1999/12684-2, período: 6/2003 a 4/2004.

FILIPPINI, J.

2004

"Biodistância entre sambaquieiros fluviais e costeiros - uma abordagem nãométrica craniana entre três sítios fluviais do vale do Ribeira - SP (Moraes, Capelinha e Pavão XVI) e três costeiros do Sul e Sudeste do Brasil (Piaçaguera, Jabuticabeira II e Tenório)", dissertação de mestrado, São Paulo, Universidade de São Paulo.

FILIPPINI, J. \& EGGERS, S.

2003 "Biodistância entre sambaquieiros fluviais e costeiros", Resumos do XII Congresso da Sociedade de Arqueologia Brasileira, São Paulo.

2005 "Testando a proximidade entre sambaquieiros fluviais (vale do Ribeira) e costeiros (litoral sul e sudeste) do Brasil", Resumos do XIII Congresso da Sociedade de Arqueologia Brasileira, Campo Grande. 
GIARDINI, L. B. \& EGGERS, S.

2003

"Variabilidade biológica entre sambaquieiros litorâneos e fluviais através de variáveis morfológicas dentárias", Resumos do XII Congresso da Sociedade de Arqueologia Brasileira, São Paulo.

2005

"Study of the biological affinity between a 9000 BP burial and the sambaqui population by discrete dental analysis", Abstracts 1st Paleopathology Association Meeting in South America, Rio de Janeiro.

HOWELLS, W. W.

1973

Cranial variation in man. Papers from the Peabody Museum of Archaeology and Ethnology 67, Cambridge, Massachusetts. Peabody Museum of Archaeology and Ethnology. Skull shapes and the map: craniometric analyses in the dispersion of modern Homo. Papers of the Peabody Museum of Archaeology and Ethnology 79, Cambridge, Massachusetts, Peabody Museum of Archaeology and Ethnology.

JOHNSON, R. A. \& WICHERN, D. W.

2002 Applied multivariate statistical analysis, Upper Saddle River, N.J., Prentice Hall.

KRONE, R.

Informaçôes ethnográficas do Valle do Ribeira do Iguape. Exploração do rio Ribeira de Iguape, Commissão Geographica e Geologica do Estado de São Paulo 2, São Paulo, Edição Typ. Brazil de Rothschield \& Co.

"As grutas calcárias do vale do Ribeira de Iguape", Revista do Instituto Geográfico e Geológico, São Paulo, vol. 8(3).

KRUG, E.

1908 O Ribeira de Iguape, São Paulo, Secretaria da Agricultura do Estado de São Paulo.

LAMING-EMPERAIRE, A.

1975 "Problèmes de préhistoire brésilienne", Annales Économies, Sociétés et Civilizations, vol. 5 .

LEONARDOS, O. H.

1938 "Concheiros naturais e sambaquis", Publicaçôes do Serviço de Fomento da Produção Mineral, avulsos, Departamento Nacional de Produção Mineral, Rio de Janeiro, vol. 37. 
Revista de Antropologia, São Paulo, USP, 2005, v. 48 no 2.

LIMA, A. P. S.

2005

Análise dos processos formativos do sitio Capelinha: estabelecimento de um contexto microrregional, dissertação de mestrado, São Paulo, Universidade de São Paulo.

LIMA, T. A.

1999-2000 "Em busca dos frutos do mar: os pescadores-coletores do litoral centro-sul do Brasil”, Revista USP, São Paulo, vol. 44, p. 270-327.

MANLY, B. F. J.

1994

Multivariate statistical methods. A primer, Londres, Chapman and Hall.

MENDONÇA, C. A. \& GOMES, M. J.

2003

"Investigaçôes geofísicas em sambaquis fluviais do vale do Ribeira", Resumos do XII Congresso da Sociedade de Arqueologia Brasileira, São Paulo.

NEVES, W. A.

1988

"Paleogenética dos grupos pré-históricos do litoral sul do Brasil (Paraná e Santa Catarina)", Pesquisas (Antropologia), vol. 43, p. 1-178.

NEVES, W. A.; HUBBE, M.; OKUMURA, M. M. M.; GONZÁLEZ-JOSÉ, R.; FIGUTI, L.; EGGERS, S.; DE BLASIS, P. A. D.

2005a "A new early Holocene human skeleton from Brazil: implications for the settlement of the New World", Journal of Human Evolution, vol. 48, p. 403-14.

NEVES, W. A.; HUBBE, M.; RIBEIRO; P. A. M.; BERNARDO, D. V.

2005b "Afinidades morfológicas de três crânios associados à Tradição Umbu: uma análise exploratória multivariada", Revista do CEPA, Santa Cruz.

PETRONE, P.

1966 "A Baixada do Ribeira, estudo de geografia humana", Geografia, São Paulo, vol. 14, Boletim no 283 .

PETRONILHO, C.; WESOLOWSKI, V.; OKUMURA, M. M. M.; EGGERS, S.

2003

"Avaliando a diversidade de estilos de vida nos sambaquis costeiros e fluviais através da paleopatologia", Resumos do XII Congresso da Sociedade de Arqueologia Brasileira, São Paulo. 
PIAZZA, W. F.

"Nota preliminar sobre o Programa Nacional de Pesquisas Arqueológicas no Estado de Santa Catarina", Programa Nacional de Pesquisas Arqueológicas - Resultados preliminares do primeiro ano 1965-1966 (Publicaçōes avulsas do Museu Paraense Emilio Goeldi), Belém, vol. 6, p. 39-46.

PROUS, A.

"Première information sur les maisons souterraines de l'Etat de São Paulo", Revista de Pré-História, São Paulo, vol. 1(1), p. 127-45.

REIS, M. J.

1980 A problemática das estruturas subterrâneas no Planalto Catarinense, dissertação de mestrado, São Paulo, Universidade de São Paulo.

ROBRAHN, E. M.

1988

A ocupação pré-colonial do vale do Ribeira de Iguape, SP: os grupos ceramistas do médio curso, dissertação de mestrado, São Paulo, Universidade de São Paulo.

ROBRAHN-GONZÁLEZ, E. M.

1999 "Diversidade cultural entre os povos ceramistas do Sul-Sudeste brasileiro: o caso do vale do Ribeira de Iguape", in TENÓRIO, M. C. (org.). Pré-História da Terra Brasilis, Rio de Janeiro, UFRJ, p. 293-306.

ROBRAHN-GONZÁLEZ, E. M. \& DE BLASIS, P. A. D.

1998

"Investigações arqueológicas no médio/baixo vale do Ribeira de Iguape, São Paulo", Revista do Museu de Arqueologia e Etnologia, São Paulo, vol. 8, p. 57-69.

ROHR, J. A.

1971

"Os sítios arqueológicos do Planalto Catarinense, Brasil", Pesquisas (Antropologia), vol. 24, p. 1-56.

SAKAI, K.

1981

Notas arqueológicas do Estado de São Paulo, Brasil, São Paulo, Instituto Paulista de Arqueologia. 
Revista de Antropologia, São Paulo, USP, 2005, v. 48 № 2.

SCHMITZ, P. I.

1996

"Visão de conjunto dos sítios de Tapera, Armação do Sul, Laranjeiras I e II, Pântano do Sul e Cabeçudas - Escavações arqueológicas do Pe. João Alfredo Rohr, SJ”, Pesquisas (Antropologia), vol. 53, p. 183-90.

SCHMITZ, P. I.; VERARDI, I.; DE MASI, M. A. N.; ROGGE, J. H.; JACOBUS, A. L. 1993

"O sítio da Praia das Laranjeiras II. Uma aldeia da tradição ceramista Itararé escavações arqueológicas do Pe. João Alfredo Rohr, SJ”, Pesquisas (Antropologia), vol. 49, p. 1-181.

TIBURTIUS, G.; BIGARELLA, I. K.; BIGARELLA, J. J.

1950-1951 "Nota prévia sobre a jazida paleontográfica de Itacoara (Joinville, Estado de Santa Catarina)", Arquivos de Biologia e Tecnologia, vol. 5/6, p. 315-40.

WARD, J. H.

1963

"Hierarchical grouping to optimize an objective function", Journal of the American Statistical Association, vol. 58, p. 236-44.

WILLEY, G. R.

An introduction to American archaeology: South America 2, Englewood Cliffs, NJ, Prentice Hall. 
ABSTRACT: Although there is no doubt about human contacts between inland and coast in Southern Brazil during archaic times, these contacts remains unknown. Due to its geomorphologic idiosyncrasies, the Ribeira de Iguape valley, in the State of São Paulo, is one of few regions in Southeastern Brazil where this communication could be easily performed. Here we present results of a craniometric analysis using 12 human skeletons from riverine shellmounds from Ribeira de Iguape valley, dated to between 6,000 and 1,200 years before present (non calibrated). These specimens were compared to 225 human skeletons from several Brazilian pre-historic sites from inland and coast. The biological affinity found in early Holocene individuals from this valley and Paleoamericans was not observed for the archaic riverine shellmound groups. No affinities were found between them and other inland series, as well. The riverine shellmound groups are close to the coastal shellmound groups from São Paulo and Paraná, which can be interpreted as evidence of a relevant contact between coast and inland, at least in Southern São Paulo State in the middle Holocene.

KEY-WORDS: craniometry, riverine shellmound, Principal Component Analysis.

Aceito em outubro de 2005. 